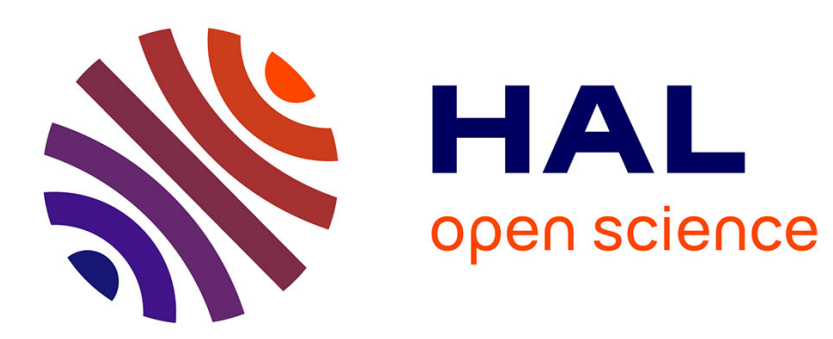

\title{
Momentum-dependent light scattering in insulating cuprates
}

\author{
F. Vernay, M. Gingras, T. Devereaux
}

\section{To cite this version:}

F. Vernay, M. Gingras, T. Devereaux. Momentum-dependent light scattering in insulating cuprates. Physical Review B: Condensed Matter and Materials Physics (1998-2015), 2007, 75 (2), 10.1103/PhysRevB.75.020403 . hal-02145561

\section{HAL Id: hal-02145561 https://hal.science/hal-02145561}

Submitted on 3 Jun 2019

HAL is a multi-disciplinary open access archive for the deposit and dissemination of scientific research documents, whether they are published or not. The documents may come from teaching and research institutions in France or abroad, or from public or private research centers.
L'archive ouverte pluridisciplinaire HAL, est destinée au dépôt et à la diffusion de documents scientifiques de niveau recherche, publiés ou non, émanant des établissements d'enseignement et de recherche français ou étrangers, des laboratoires publics ou privés. 


\title{
Momentum-dependent light scattering in insulating cuprates
}

\author{
F. H. Vernay, ${ }^{1,2}$ M. J. P. Gingras, ${ }^{1,3}$ and T. P. Devereaux ${ }^{1,2}$ \\ ${ }^{1}$ Department of Physics and Astronomy, University of Waterloo, Waterloo, Ontario, Canada N2L $3 G 1$ \\ ${ }^{2}$ Pacific Institute for Theoretical Physics, University of British Columbia, Vancouver, British Columbia, Canada V6T 1Z1 \\ ${ }^{3}$ Department of Physics and Astronomy, University of Canterbury, Christchurch 8020, New Zealand \\ (Received 26 May 2006; revised manuscript received 2 October 2006; published 19 January 2007)
}

\begin{abstract}
We investigate the problem of inelastic $\mathrm{x}$-ray scattering in the spin-1/2 Heisenberg model on the square lattice. We first derive a momentum-dependent scattering operator for the $A_{1 g}$ and $B_{1 g}$ polarization geometries. On the basis of a spin-wave analysis, including magnon-magnon interactions and exact diagonalizations, we determine the qualitative shape of the spectra. We argue that our results may be relevant to help interpret inelastic x-ray scattering experiments in the antiferromagnetic phase of the cuprates.
\end{abstract}

DOI: 10.1103/PhysRevB.75.020403

The advances made in third-generation light sources have recently provided new insights into the study of electron dynamics in strongly correlated systems via resonant inelastic $\mathrm{x}$-ray scattering (RIXS). Detailed information has already been obtained on Mott gap excitations and orbital transitions as a function of doping in the cuprate families. ${ }^{1-6}$ However, due to limitations in resolution, $\sim 300 \mathrm{meV}$ near the elastic line, excitations at low energies remain hidden. On the other hand, low-energy excitations at small photon momentum transfers, e.g., phonons, magnons, and electron-hole excitations in metals and superconductors, have been well characterized via Raman spectroscopy. ${ }^{7}$ Ultimately, achieving a detailed understanding of the momentum and polarization dependence of low-energy excitations in strongly correlated matter would greatly clarify the interplay between various degrees of freedom, such as antiferromagnetism, chargedensity-wave order, and superconductivity, often present in system with many competing interactions. ${ }^{8}$

In the energy range below a few hundred meV lies one of the most prominent features observed via Raman scattering in Heisenberg antiferromagnets-the two-magnon feature at energy $\sim 2.7 J$, with $J$ the nearest-neighbor magnetic exchange. While the peak frequency of the broad two-magnon peak in Raman scattering is well understood, the asymmetry of the line shape as well as the polarization dependence remain unexplained, even though it has been lavished with attention. ${ }^{9}$ Preliminary RIXS studies on the $\mathrm{Cu} K$ edge in $\mathrm{LaCuO}_{4}$ (Ref. 10) and $M$ edge in $\mathrm{CaCuO}_{2}$ (Ref. 11) have shown evidence for low-energy two-magnon scattering. Since in the near future this low-energy window will open for inelastic x-ray studies, it will afford an opportunity to study the dynamics of magnon excitations via the charge degrees of freedom that will complement neutron and Raman scattering studies.

Lorenzana and Sawatzky ${ }^{12}$ investigated the properties of phonon-assisted absorption in two-dimensional (2D) Heisenberg antiferromagnets, and investigated the bimagnon spectrum probed by infrared conductivity. In this paper we extend these calculations to present a theory of inelastic x-ray scattering of the two-magnon response in a Heisenberg antiferromagnet. In particular we show that the momentum and polarization dependence can provide detailed information on the nature of magnon-magnon interactions in the parent insulating cuprate compounds.

We remark at the outset that we neglect specific resonant
PACS number(s): 75.25.+z, 72.10.Di, 78.70.Ck, 72.80.Ga

matrix elements relevant to the RIXS process including transitions resulting from the creation of the core hole, and work in the restricted model of the half-filled single-band Hubbard model on the square lattice to capture scattering via magnon creation. Thus we neglect specific pathways of charge excitation, such as the $\mathrm{Cu} K$ edge $1 s-4 p$ transition, and how double spin flips may occur near the site where the core hole is created. Such kinematic details are indeed important to determine accurately the RIXS intensity as well as the proper symmetry and polarization of spin excitations that may be probed by specific $x$-ray transitions. However, in order to obtain a preliminary understanding of how the two-magnon response can be probed and how polarization may enter, we first simply focus on the evolution of the two-magnon response, far from any specific resonance, for nonzero photon momentum transfers $\mathbf{q}$.

Transitions via light scattering can be created via dipole or multipole matrix elements involving states within the conduction band or out of the valence band. These transitions may be selected by orienting incident and scattering polarization light vectors, $\hat{\mathbf{e}}_{i}$ and $\hat{\mathbf{e}}_{f}$, respectively. The photons entering in the scattering process are represented by $\left(\omega_{i, f}, \mathbf{k}_{i, f}, \hat{\mathbf{e}}_{i, f}\right)$ where the indices $i$ and $f$ represent the incoming and outgoing photon, respectively. Since we are interested in the insulating phase, the scattering of light is induced by the interband part of the operator. Following Refs. 13 and 14, we derive a finite momentum transfer $\mathbf{q}$, $\mathbf{q} \equiv \mathbf{k}_{i}-\mathbf{k}_{f}$, scattering operator for different polarization geometries. The interband part of the scattering matrix element is given by

$$
\begin{aligned}
\left\langle f\left|M_{r}\right| i\right\rangle= & \sum_{\nu}\left(\frac{\left\langle f\left|\mathbf{J}_{k_{f}} \cdot \hat{\mathbf{e}}_{f}\right| \nu\right\rangle\left\langle\nu\left|\mathbf{J}_{-\mathbf{k}_{i}} \cdot \hat{\mathbf{e}}_{i}\right| i\right\rangle}{\epsilon_{\nu}-\epsilon_{i}-\omega_{i}}\right. \\
& \left.+\frac{\left\langle f\left|\mathbf{J}_{-\mathbf{k}_{i}} \cdot \hat{\mathbf{e}}_{i}\right| \nu\right\rangle\left\langle\nu\left|\mathbf{J}_{\mathbf{k}_{f}} \cdot \hat{\mathbf{e}}_{f}\right| i\right\rangle}{\epsilon_{\nu}-\epsilon_{i}+\omega_{f}}\right)
\end{aligned}
$$

where $\nu$ represents states out of the lower Hubbard band, and $\mathbf{J}_{\mathbf{k}}$ is the current operator $\mathbf{J}_{\mathbf{k}}=\Sigma_{\mathbf{p}, \sigma}\left(\partial \epsilon_{\mathbf{p}} / \partial \mathbf{p}\right) c_{\mathbf{p}+k / 2, \sigma}^{\dagger} c_{\mathbf{p}-k / 2, \sigma}$, with $\epsilon_{\mathbf{p}}=-2 t\left[\cos \left(p_{x} a\right)+\cos \left(p_{y} a\right)\right]$ for a square lattice with lattice constant $a$, and nearest-neighbor hopping $t$. The current may be expressed as 


$$
\begin{aligned}
\mathbf{J}_{\mathbf{k}_{f}} \cdot \hat{\mathbf{e}}_{f} & =i t \sum_{\mathbf{r}, \boldsymbol{\delta}, \sigma} \hat{e}_{f}^{\boldsymbol{\delta}} e^{-i \mathbf{k}_{f}} \cdot(\mathbf{r}+\boldsymbol{\delta} / 2)\left[c_{\mathbf{r}+\boldsymbol{\delta}}^{\dagger} c_{\mathbf{r}, \sigma}-c_{\mathbf{r}, \sigma}^{\dagger} c_{\mathbf{r}+\boldsymbol{\delta}, \sigma}\right] \\
& \equiv \sum_{\mathbf{r}, \boldsymbol{\delta}} \hat{e}_{f}^{\boldsymbol{\delta}} J_{\mathbf{k}_{f}}(\mathbf{r}, \boldsymbol{\delta})
\end{aligned}
$$

where $\hat{e}_{f}^{\boldsymbol{\delta}}$ is the projection of the polarization vector along the neighbor direction $\boldsymbol{\delta}$. In the insulating state, $|i\rangle$ and $|f\rangle$ are singly occupied states, whereas $|\nu\rangle$ are excited states consisting of a doubly occupied state at site $\mathbf{r}+\boldsymbol{\delta}$. Thus the energy of the excited state is roughly given by $\epsilon_{\nu} \approx U+\epsilon_{i}$, and the intermediate states may be collapsed using the identity $\frac{1}{4}$ $-\mathbf{S}_{i} \cdot \mathbf{S}_{j}=\frac{1}{2} c_{i, \sigma^{\prime}}^{\dagger} c_{j, \sigma} c_{j, \sigma^{\prime}}^{\dagger} c_{i, \sigma^{\prime}}$, which simply means that the exchange process (and therefore a doubly occupied site) is allowed only if spins $\sigma$ and $\sigma^{\prime}$ are opposite. ${ }^{14}$ Finally, we recast $\left\langle f\left|M_{r}\right| i\right\rangle$ in Eq. (1) via the following scattering operator:

$$
\begin{aligned}
\mathcal{O}(\mathbf{q})= & 8 t^{2} \sum_{\mathbf{r}, \boldsymbol{\delta}} e^{i \mathbf{q} \cdot(\mathbf{r}+\boldsymbol{\delta} / 2)}\left(\hat{\boldsymbol{\delta}} \cdot \hat{\mathbf{e}}_{f}\right)\left(\hat{\boldsymbol{\delta}} \cdot \hat{\mathbf{e}}_{i}\right)\left(\frac{1}{4}-\mathbf{S}_{\mathbf{r}} \cdot \mathbf{S}_{\mathbf{r}+\boldsymbol{\delta}}\right)\left(\frac{1}{U+\omega_{f}}\right. \\
& \left.+\frac{1}{U-\omega_{i}}\right) .
\end{aligned}
$$

For crossed polarizations, $\hat{\mathbf{e}}_{i, f}=(\hat{x} \pm \hat{y}) / \sqrt{2}$, transforming as $B_{1 g}$, and for parallel polarizations along $\hat{x}$ and $\hat{y}$, transforming as $A_{1 g}$, we obtain the following expression:

$$
\begin{aligned}
\mathcal{O}_{A_{1 g}, B}(\mathbf{q})= & -8 t^{2}\left(\frac{1}{U+\omega_{f}}+\frac{1}{U-\omega_{i}}\right) \sum_{i, \boldsymbol{\delta}} P_{A 1, B 1}(\boldsymbol{\delta}) \\
& \times\left[\mathbf{S}_{i} \cdot \mathbf{S}_{i+\boldsymbol{\delta}}\right] \cos \left(\mathbf{q} \cdot \mathbf{r}_{i}+\mathbf{q} \cdot \boldsymbol{\delta} / 2\right),
\end{aligned}
$$

with $P_{A 1, B 1}(\boldsymbol{\delta})=1$, for $\boldsymbol{\delta}=a \hat{\boldsymbol{x}}$, and $1,-1$ for $\boldsymbol{\delta}=a \hat{\boldsymbol{y}}$ for $A_{1 g}$ and $B_{1 g}$, respectively. This is a finite-momentum generalization of the usual Loudon-Fleury light scattering operator, and for $\mathbf{q}=\mathbf{0}$ the above expressions give the standard Raman results. ${ }^{13,14}$ We note that in general subtractions of spectra for different polarization orientations are needed in order to fully extract symmetry-deconvolved spectra. ${ }^{7}$

Henceforth, we restrict ourselves to the half-filled Hubbard model with $t / U$ small, and focus on its spin- $\frac{1}{2}$ antiferromagnetic Heisenberg representation with Hamiltonian $\mathcal{H}$. We first investigate the q-dependent inelastic response via the spin-wave (SW) approximation. We proceed as usual $^{9,14-20}$ and express $\mathcal{H}$ in its $\mathrm{SW}$ representation, $\mathcal{H}_{\mathrm{SW}}=C s t+\sum_{\mathbf{k}} \omega_{\mathbf{k}}\left(\alpha_{\mathbf{k}}^{\dagger} \alpha_{\mathbf{k}}+\beta_{\mathbf{k}}^{\dagger} \beta_{\mathbf{k}}\right)$, with $\omega_{\mathbf{k}}=J S Z \sqrt{1-\gamma_{\mathbf{k}}^{2}}$ the magnon dispersion, and $2 u_{\mathbf{k}}^{2}-1=2 v_{\mathbf{k}}^{2}+1=1 / \sqrt{1-\gamma_{\mathbf{k}}^{2}}$ and $\gamma_{\mathbf{k}}=(1 / 2)\left[\cos \left(k_{x} a\right)+\cos \left(k_{y} a\right)\right]$. Performing the same transformations to the scattering operators $\mathcal{O}_{A_{1 g}, B_{1 g}}$ we obtain:

$$
\begin{aligned}
\mathcal{O}_{A_{1 g}, B_{1 g}} \propto & \sum_{\mathbf{k}} \beta_{\mathbf{k}}^{\dagger} \alpha_{\mathbf{k}+\mathbf{q}}^{\dagger}\left\{-\left[\cos \left(\frac{q_{x} a}{2}\right) \pm \cos \left(\frac{q_{y} a}{2}\right)\right]\right. \\
& \times\left(u_{\mathbf{k}} v_{\mathbf{k}+\mathbf{q}}+v_{\mathbf{k}} u_{\mathbf{k}+\mathbf{q}}\right)+\left(u_{\mathbf{k}} u_{\mathbf{k}+\mathbf{q}}+v_{\mathbf{k}} v_{\mathbf{k}+\mathbf{q}}\right) \\
& \left.\times\left[\cos \left(k_{x} a\right) \cos \left(\frac{q_{x} a}{2}\right) \pm \cos \left(k_{y} a\right) \cos \left(\frac{q_{y} a}{2}\right)\right]\right\},
\end{aligned}
$$

where we have neglected the prefactor in Eq. (4). For $A_{1 g}$ and $\mathbf{q}=0$, we recover the familiar form of the Raman operator which, being proportional to $\mathcal{H}$, commutes with $\mathcal{H}$, giving no Raman scattering in that channel.

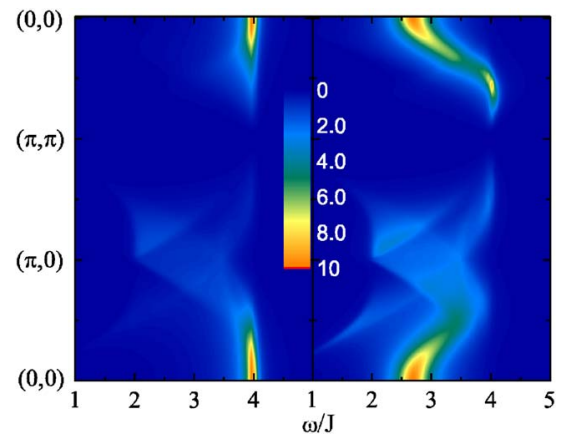

FIG. 1. (Color online) Spin-wave intensity spectrum without $\left[I_{0}(\mathbf{q}, \omega)\right.$, left $]$ and with $\left[I_{\mathrm{RPA}}(\mathbf{q}, \omega)\right.$, right $]$ magnon-magnon interaction. The color intensity scale is 2.2 times smaller for the right panel than the left.

One also finds that $\mathcal{O}_{A_{1 g}, B_{1 g}}$ vanish for $\mathbf{q}=\mathbf{Q}=(\pi, \pi)$ and symmetry-related points for both $A_{1 g}$ and $B_{1 g}$. This is a consequence of including only nearest-neighbor spin interactions in the Heisenberg model. ${ }^{21}$ While in our case the response vanishes for the antiferromagnetic reciprocal lattice vectors, if one includes longer-range interactions, then this restriction may be lifted. ${ }^{19}$ Thus the $\mathrm{x}$-ray Raman response for these wave vectors may provide a window to sample the role of longer-range spin interactions.

The scattering intensity is proportional to $\left\langle f\left|\mathcal{O}_{B_{1 g}}\right| i\right\rangle^{2}$, while satisfying energy conservation $\omega=\omega_{\mathbf{k}}+\omega_{\mathbf{k}+\mathbf{q}}$. We first focus on the $B_{1 g}$ channel, where the two-magnon scattering is most prominent for $\mathbf{q}=0$. The left panel of Fig. 1 shows the $B_{1 g}$ bare scattering intensity, neglecting magnon-magnon interactions: $I_{0}(\mathbf{q}, \omega)=(1 / 2 N) \Sigma_{\mathbf{k}} \delta\left(\omega_{\mathbf{k}}+\omega_{\mathbf{k}+\mathbf{q}}-\omega\right) b_{\mathbf{k}, \mathbf{q}}^{2}$, where $b_{\mathbf{k}, \mathbf{q}}$ is the term in curly brackets in Eq. (5). The $I_{0}(\mathbf{q}=0, \omega)$ intensity recovers the standard Raman response, which for the noninteracting case has a peak at $\omega=4 J$ due to the large magnon density of states at $\mathbf{k}=(\pi, 0)$ projected out by the $B_{1 g}$ operator. For nonzero q, two magnons are created with wave vectors $\mathbf{k}$ of different magnitude and direction, leaving behind a reorganized spin configuration, and the response is given by convolving two magnon densities of states at different $\mathbf{k}$ separated by $\mathbf{q}$. We note that, while the response is still governed by $\sim 4 J$ for finite $\mathbf{q}$ due to the magnon density of states being largest at $\mathbf{k}=(\pi, 0)$, the intensity is highly suppressed as $\mathbf{q}$ approaches $\mathbf{Q}$.

Magnon-magnon interactions need to be included to describe more realistically the local spin rearrangement. ${ }^{9,14-20}$ In particular, interactions lead to a reduction of the peak frequency for $B_{1 g}$ Raman scattering to $2.78 J$, where $J$, as shown by Singh et al., ${ }^{22}$ is also renormalized by quantum corrections. Many diagrams contribute to the magnonmagnon vertex corrections, and a subset has been investigated for $\mathbf{q}=0$ Raman scattering. ${ }^{19}$ In that case, magnonmagnon interactions lower the relevant energy scale from $4 \mathrm{~J}$ to $3 J$ due to the local breaking of six exchange bonds for two neighboring spin flips. For finite q considered here, the two magnons are created with net momentum q, which distributes the spin flips over longer length scales. Since magnonmagnon vertex corrections are expected to weaken as the spin arrangement occurs at larger length scales, we approximate the renormalized response by a generalized random- 
phase approximation (RPA) form $I_{\mathrm{RPA}}(\mathbf{q}, \omega) \sim I_{0}(\mathbf{q}, \omega) /[1$ $\left.+J(\mathbf{q}) I_{0}(\mathbf{q}, \omega)\right]$, where the function $J(\mathbf{q})=\left[\cos \left(q_{x} a / 2\right)\right.$ $\left.+\cos \left(q_{y} a / 2\right)\right] / 2$ is taken to recover the Raman form at $\mathbf{q}$ $=0$ and properties of the solution to the Bethe-Salpeter equation for finite q. A proper treatment of magnon-magnon interactions for all $\mathbf{q}$ in the SW framework is a topic of future research. As shown in the right panel of Fig. 1, the magnon interactions for larger $\mathbf{q}$ bring the peak down to $\omega=2.78 \mathrm{~J}$ for $B_{1 g} \mathbf{q}=0$ Raman scattering, but the general weakening of the magnon interactions at larger $\mathbf{q}$ gives back the unrenormalized response at larger $\mathbf{q}$ with, in particular, the response still vanishing at $\mathbf{q}=\mathbf{Q}$. On the other hand, the dispersion of the peak changes dramatically when magnon-magnon interactions are included, where the peak hardens at finite $\mathbf{q}$ from the $\Gamma$ point, $\mathbf{q}=0$.

The results above differ slightly from those obtained in Ref. 12 for infrared absorption from multimagnons. While the dispersion of the peaks is similar to our results, Ref. 12 found a bimagnon response which is dominated by a sharp peak with a small bimagnon lifetime for momentum transfers $(\pi, 0)$. Since the form factors are different for infrared and polarized x-ray Raman measurements, the bimagnon spectrum has different projections for the results presented here, favoring $\mathbf{q}=0$ for a bimagnon in the $B_{1 g}$ channel, and a growth of the $A_{1 g}$ component with increasing $q$. Thus the $A_{1 g}$ response is more similar to the infrared one, as it weights out similar regions in the magnetic Brillouin zone (BZ). We note that the phonon-assisted bimagnon response for X-ray Raman scattering, considered in the same vein as Ref. 12, would also have similar polarization-dependent form factors as those considered here, coming from the symmetry classification of the active phonon modes involved in the scattering.

In order to explore the semiquantitative validity of the SW and RPA results, we investigate the q-dependent inelastic x-ray scattering for the Heisenberg spin Hamiltonian $\mathcal{H}$, using an exact diagonalization approach, considering both the $A_{1 g}$ and $B_{1 g}$ channels. Although exact diagonalization is limited because of the prohibitive size of the Hilbert space, Gagliano and Balseiro ${ }^{23}$ demonstrated that it is a powerful technique that allows one to compute the dynamical quantities easily. Noteworthy in the context of numerical investigations of Raman spectra, Sandvik et al. ${ }^{15}$ showed that, even though the spectra obtained by the Lanczos method are extremely size dependent, they are of direct relevance to test the response calculated from a spin-wave analysis. As in Ref. 24 , the spectra are evaluated by computing the continued fraction

$$
I(\mathbf{q}, \omega)=-\frac{1}{\pi} \operatorname{Im}\left\langle\Psi_{\text {g.s. }}\left|\mathcal{O}^{\dagger}(-\mathbf{q}) \frac{1}{z-\mathcal{H}} \mathcal{O}(\mathbf{q})\right| \Psi_{\text {g.s. }}\right\rangle
$$

with $z=\omega+i \epsilon+E_{0}$, where $E_{0}$ is the ground-state energy and $\epsilon$ is a damping factor.

We first compute the ground-state energy. For $N=16$ sites we have $E_{0} /(N J) \approx-0.70178020$ and wave vector $\left|\Psi_{\text {g.s. }}\right\rangle$. We then begin evaluating the continued fraction with the starting state

$$
|\Phi(\mathbf{q})\rangle=\frac{\mathcal{O}(\mathbf{q})\left|\Psi_{\text {g.s. }}\right\rangle}{\sqrt{\left\langle\Psi_{\text {g.s. }}\left|\mathcal{O}^{\dagger}(-\mathbf{q}) \mathcal{O}(\mathbf{q})\right| \Psi_{\text {g.s. }}\right\rangle}} .
$$

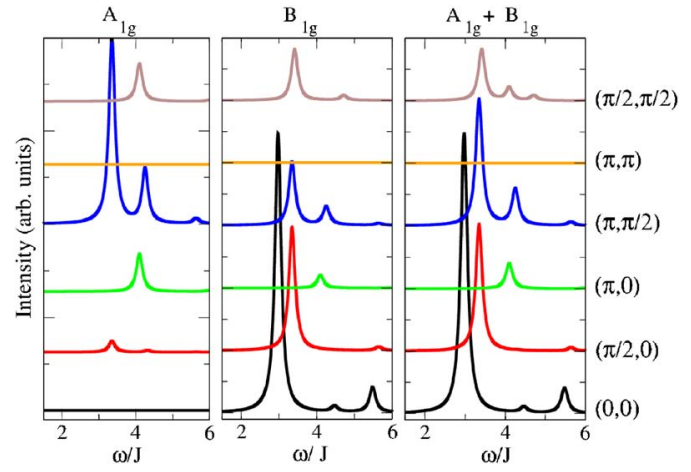

FIG. 2. (Color online) 16-site cluster: exact diagonalizations for $A_{1 g}$ and $B_{1 g}$ polarizations, to be compared with spin-wave results. The spectra are shown for the independent momenta $\mathbf{q}$ allowed for the $N=16$-site cluster (given on the side of the rightmost panel). The relative intensity of the curves is given by $\left\langle\mathcal{O}(\mathbf{q})^{2}\right\rangle$.

Here, compared to Raman scattering, care has to be taken in computing Eq. (6): the state $|\Phi(\mathbf{q})\rangle$ being in a different subspace at nonzero $\mathbf{q}$ than the ground state, the matrix elements of $\mathcal{H}$ to compute the continued fraction have to be expressed in the corresponding subspace. The results are summarized in Figs. 2 and 3.

For the case of $B_{1 g}$ scattering, we recover prior results for $\mathbf{q}=0,{ }^{14}$ while we see that the peak disperses to higher energies, approaching $4 J$ both for $\mathbf{q}$ along the BZ diagonal as well as along the axes. In light of the RPA results in the right panel of Fig. 1, we attribute this dispersion to a weakening of magnon interactions at larger $\mathbf{q}$. At the same time, the overall intensity diminishes for larger $\mathbf{q}$ due to the cosine form factor appearing in Eq. (4).

Since exact diagonalizations deal with small clusters, it is very difficult to make a quantitative finite-size scaling analysis for the spectral shape. ${ }^{15}$ Therefore we repeat the calculation for the $B_{1 g}$ polarization with a 20 -site cluster, and plot the results in Fig. 3. It is well known that the 16-site cluster has additional symmetries ${ }^{25}$ (hypercubelike) and it is therefore important to check for similar $\mathbf{q}$ points in the 20-site cluster. Due to the different shape and boundary conditions of this cluster, we are not able to explore the same momenta in the BZ. However direct inspection of the $\mathbf{q}$ points $(0,0)$, $(\pi, 0)$, and $(\pi, \pi)$ reveals that many of the features are qualitatively similar: the $\mathbf{q}=\mathbf{0}$ peak is at lower frequency than $(\pi, 0)$, and the intensity weakens at finite $q \equiv|\mathbf{q}|$ as in the

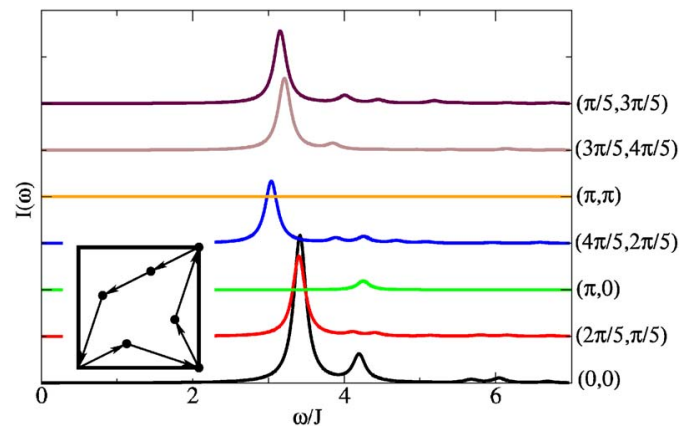

FIG. 3. (Color online) 20-site cluster: exact diagonalizations in the $B_{1 g}$ channel. The inset is a schematic of the investigated $\mathbf{q}$ points. 
16-site case (compare with the middle panel of Fig. 2). In addition, the dispersion along the path in the $\mathbf{q}$ space is of the same order $\sim J$. In the RPA calculation, the minimum frequency of the main peak is at $(0,0)$. For the compatible $\mathbf{q}$ points, the results are consistent with the RPA approach as well (same qualitative dispersion and minimum). We therefore believe that exact diagonalizations give a correct qualitative description of the response.

For $A_{1 g}$, a peak appears for finite $\mathbf{q} \cdot{ }^{21}$ Generally the peak disperses toward $4 J$ for larger $\mathbf{q}$, following essentially the $B_{1 g}$ spectrum. The $B_{1 g}$ intensity falls with increasing $\mathbf{q}$ while at the same time the $A_{1 g}$ spectral intensity grows. In fact, we note that the intensity for $A_{1 g}$ approaches that of the $B_{1 g}$ spectrum for $\mathbf{q}=(\pi, \pi / 2)$, where the scattering operators in Eq. (4) are the same.

Thus, the overall intensity in an unpolarized measurement, given by $I_{A_{1 g}}+I_{B_{1 g}}$ as shown in Fig. 2, is dominated by the $B_{1 g}$ channel for small $\mathbf{q}$, and a mixture of $A_{1 g}$ and $B_{1 g}$ for larger $\mathbf{q} .{ }^{26}$ The main results are thus that for small $\mathbf{q}$, the two-magnon Raman peak rapidly disperses upward in energy, for $\mathbf{q}$ both along the BZ diagonal and BZ axes. At the same time, a contribution from the $A_{1 g}$ channel develops, as the intensity of the $B_{1 g}$ contribution falls with increasing $\mathbf{q}$. We note, however, that the problem of describing the twomagnon profile for $\mathbf{q}=0$, which includes the differences between $A_{1 g}$ and $B_{1 g}$ intensities, as well as the width of the line shapes, remains an issue here as well (see, e.g., Ref. 9). Thus more detailed treatments, including further spin-spin interactions, ring exchange, and possibly spin-phonon coupling, would need to be incorporated in low-energy inelastic x-ray scattering as well. Besides affecting the overall line shape, the behavior at different momentum points, such as the special point at $(\pi, \pi)$, may change. Thus, the detailed momen- tum dependence of the spectra may be able to provide important information of the types and extensions of spin interactions in antiferromagnets.

Finally we remark on our results in terms of the current capabilities of RIXS experiments. Empirically, from fitting the two-magnon position and spin-wave velocity, $J$ in the cuprates is estimated as $J \approx 0.13 \mathrm{eV}$. Thus the two-magnon Raman peak would lie generally obscured in the elastic line at currently available resolution, which is several orders of magnitude larger than the inelastic contribution. It is a possibility that the two-magnon contribution would emerge from underneath the elastic line at larger momentum transfers $\mathbf{q}$ as the peak in X-ray Raman scattering disperses to $4 \mathrm{~J}$. If the energy resolution can be enhanced, we propose that perhaps a clear two-magnon contribution will become visible. However, a proper treatment of the resonant matrix elements needs to be considered, which, while not affecting the dispersion, may change the relative intensity of the spectra at specific q. This remains a topic for future research.

Note added in proof. Recently, we became aware of a closely related preprint. ${ }^{27}$ Our results are similar for small momenta but they differ for $\mathbf{q}=(\pi, \pi)$ due to a slight difference in the form of their scattering operator.

We acknowledge important discussions with B. Freelon, M. Greven, M. Z. Hasan, J. P. Hill, Y.-J. Kim, M. V. Klein, S. Maekawa, G. Sawatzky, K. M. Shen, Z.-X. Shen, and R. R. P. Singh. Partial support for this work was provided by NSERC of Canada, the Canada Research Chair Program (Tier I) (M.G), the Province of Ontario (M.G.), the Alexander von Humboldt Foundation (T.P.D.), and ONR Grant No. N00014-05-1-0127 (T.P.D.). M.G. acknowledges support from the Canadian Institute for Advanced Research.
${ }^{1}$ L. Lu, X. Zhao, J. N. Hancock, G. Chabot-Couture, N. Kaneko, O. P. Vajk, G. Yu, S. Grenier, Y. J. Kim, D. Casa, T. Gog, and M. Greven, Phys. Rev. Lett. 95, 217003 (2005).

${ }^{2}$ P. Abbamonte, C. A. Burns, E. D. Isaacs, P. M. Platzman, L. L. Miller, S. W. Cheong, and M. V. Klein, Phys. Rev. Lett. 83, 860 (1999).

${ }^{3}$ M. Z. Hasan, E. D. Isaacs, Z.-X. Shen, L. L. Miller, K. Tsutsui, T. Tohyama, and S. Maekawa, Science 288, 1811 (2000).

${ }^{4}$ Y. J. Kim, J. P. Hill, C. A. Burns, S. Wakimoto, R. J. Birgeneau, D. Casa, T. Gog, and C. T. Venkataraman, Phys. Rev. Lett. 89, 177003 (2002).

${ }^{5}$ Y. J. Kim, J. P. Hill, G. D. Gu, F. C. Chou, S. Wakimoto, R. J. Birgeneau, Seiki Komiya, Yoichi Ando, N. Motoyama, K. M. Kojima, S. Uchida, D. Casa, and T. Gog, Phys. Rev. B 70, 205128 (2004).

${ }^{6}$ A. Kotani and S. Shin, Rev. Mod. Phys. 73, 203 (2001).

${ }^{7}$ T. P. Devereaux and R. Hackl, cond-mat/0607554; Rev. Mod. Phys. (to be published).

${ }^{8}$ E. Dagotto, Science 309, 257 (2005).

${ }^{9}$ P. J. Freitas and R. R. P. Singh, Phys. Rev. B 62, 5525 (2000), and references therein.

${ }^{10}$ J. P. Hill and Y.-J. Kim (private communication).

${ }^{11}$ B. Freelon et al. (unpublished).

${ }^{12}$ J. Lorenzana and G. A. Sawatzky, Phys. Rev. Lett. 74, 1867 (1995); Phys. Rev. B 52, 9576 (1995)
${ }^{13}$ P. A. Fleury and R. Loudon, Phys. Rev. 166, 514 (1968)

${ }^{14}$ B. S. Shastry and B. I. Shraiman, Int. J. Mod. Phys. B 5, 365 (1991); Phys. Rev. Lett. 65, 1068 (1990).

${ }^{15}$ A. W. Sandvik, S. Capponi, D. Poilblanc, and E. Dagotto, Phys. Rev. B 57, 8478 (1998)

${ }^{16}$ V. Yu. Irkhin, A. A. Katanin, and M. I. Katsnelson, Phys. Rev. B 60, 1082 (1999).

${ }^{17}$ A. A. Katanin and A. P. Kampf, Phys. Rev. B 67, 100404(R) (2003).

${ }^{18}$ R. W. Davies, S. R. Chinn, and H. J. Zeiger, Phys. Rev. B 4, 992 (1971).

${ }^{19}$ C. M. Canali and S. M. Girvin, Phys. Rev. B 45, 7127 (1992).

${ }^{20}$ A. V. Chubukov and D. M. Frenkel, Phys. Rev. B 52, 9760 (1995).

${ }^{21}$ J. van den Brink (private communication).

${ }^{22}$ R. R. P. Singh, P. A. Fleury, K. B. Lyons, and P. E. Sulewski, Phys. Rev. Lett. 62, 2736 (1989).

${ }^{23}$ E. R. Gagliano and C. A. Balseiro, Phys. Rev. Lett. 59, 2999 (1987).

${ }^{24}$ Elbio Dagotto, Rev. Mod. Phys. 66, 763 (1994).

${ }^{25}$ Y. Hasegawa and D. Poilblanc, Phys. Rev. B 40, 9035 (1989).

${ }^{26}$ Here we neglect contributions from the $B_{2 g}$ channel, which are identically zero for nearest-neighbor spin interaction.

${ }^{27}$ A. Donkov and A. V. Chubukov cond-mat/0609002. 